\title{
Autologous Genetically-modified MAGE-A4 C1032 CD8alpha T Cells
}

National Cancer Institute

\section{Source}

National Cancer Institute. Autologous Genetically-modified MAGE-A4 C1032 CD8alpha T

Cells. NCI Thesaurus. Code C162804.

Autologous human T-lymphocytes transduced with a retroviral vector encoding a T-cell receptor (TCR) specific for the human melanoma antigen A4 (MAGE-A4) and the CD8alpha co-receptor, with potential immunostimulatory and antineoplastic activities. Upon leukapheresis, isolation, transduction, expansion ex vivo, and reintroduction into the patient, the autologous genetically-modified MAGE-A4 C1032 CD8alpha T-cells bind to tumor cells expressing MAGE-A4. This may result in both inhibition of growth and increased cell death of MAGE-A4-expressing tumor cells. The tumor-associated antigen MAGE-A4, a member of the MAGE-A family of cancer testis antigens, is overexpressed by a variety of cancer cell types. Co-expression of CD8alpha may broaden the immune response against tumors and increase antitumor activity by converting CD4+ helper Tcells into CD8+ cytotoxic T-cells. 